\title{
Dopamine, Time, and Impulsivity in Humans
}

\author{
Alex Pine, ${ }^{1,2}$ Tamara Shiner, ${ }^{1}$ Ben Seymour, ${ }^{1,3,4}$ and Raymond J. Dolan ${ }^{1}$ \\ ${ }^{1}$ Wellcome Trust Centre for Neuroimaging, London WC1N 3BG, United Kingdom, ${ }^{2}$ Clinical Psychopharmacology Unit, Sub-Department of Clinical Health \\ Psychology and ${ }^{3}$ Economic and Social Research Council Centre for Economic Learning and Social Evolution, University College London, London WC1E \\ 6BT, United Kingdom, and 4Department of Clinical Neurology, Addenbrookes Hospital, Cambridge CB2 OQQ, United Kingdom
}

Disordered dopamine neurotransmission is implicated in mediating impulsiveness across a range of behaviors and disorders including addiction, compulsive gambling, attention-deficit/hyperactivity disorder, and dopamine dysregulation syndrome. Whereas existing theories of dopamine function highlight mechanisms based on aberrant reward learning or behavioral disinhibition, they do not offer an adequate account of the pathological hypersensitivity to temporal delay that forms a crucial behavioral phenotype seen in these disorders. Here we provide evidence that a role for dopamine in controlling the relationship between the timing of future rewards and their subjective value can bridge this explanatory gap. Using an intertemporal choice task, we demonstrate that pharmacologically enhancing dopamine activity increases impulsivity by enhancing the diminutive influence of increasing delay on reward value (temporal discounting) and its corresponding neural representation in the striatum. This leads to a state of excessive discounting of temporally distant, relative to sooner, rewards. Thus our findings reveal a novel mechanism by which dopamine influences human decision-making that can account for behavioral aberrations associated with a hyperfunctioning dopamine system.

\section{Introduction}

The characteristic loss of self-control and impulsivity associated with aberrant dopamine function is exemplified by disorders such as addiction, attention-deficit/hyperactivity disorder (ADHD), and dopamine dysregulation syndrome (Winstanley et al., 2006; Dagher and Robbins, 2009; O'Sullivan et al., 2009). In the latter, dopamine replacement therapy in the treatment of Parkinson's disease (PD) renders some patients prone to compulsive behavior, which manifests itself as excess gambling, shopping, eating, and other shortsighted behaviors. However, the broad phenotype of impulsivity that characterizes these behaviors subsumes a diversity of distinct decision-making processes that can be dissociated neurobiologically and pharmacologically (Evenden, 1999; Ho et al., 1999; Winstanley et al., 2004a, 2006; Dalley et al., 2008). These include a lack of inhibition of prepotent motor responses, overweighting of rewards relative to losses, failure to slow down in the face of decisionconflict, and a propensity to choose smaller-sooner over larger-later rewards.

In principle, some of the aforementioned deficits can be related to dopaminergic effects by way of dopamine's established role in reward learning (Redish, 2004; Frank et al., 2007; Dagher and Robbins, 2009). However, temporal (or choice) impulsivitythe preference for smaller-sooner over larger-later rewards,

Received Dec. 6, 2009; revised April 18, 2010; accepted April 22, 2010.

This work was funded by a Wellcome Trust Programme Grant to R.J.D., and A.P. was supported by a Medical Research Council studentship. We thank K. Friston, J. Roiser, and V. Curran for help with planning and analyses, and for insightful discussions.

Correspondence should be addressed to Alex Pine, Wellcome Trust Centre for Neuroimaging, 12 Queen Square, London WC1N 3BG, United Kingdom. E-mail: a.pine@ucl.ac.uk.

DOI:10.1523/JNEUROSCI.6028-09.2010

Copyright $\odot 2010$ the authors $\quad 0270-6474 / 10 / 308888-09 \$ 15.00 / 0$ due to excessive discounting of future rewards (Ainslie, 1975; Evenden, 1999; Ho et al., 1999; Cardinal et al., 2004)—is much harder to account for in terms of learning, although it remains an important feature of putative dopaminergic impulsivity. Indeed, laboratory tests of intertemporal choice indicate that addicts and a subgroup of ADHD patients appear to have abnormally high temporal discount rates, strongly preferring smaller-sooner rewards (Sagvolden and Sergeant, 1998; Bickel and Marsch, 2001; Solanto et al., 2001; Winstanley et al., 2006; Bickel et al., 2007). This poses the question of whether dopamine has a specific role in computing how the temporal proximity of a reward relates to its subjective value (i.e., rate of temporal discounting), independent of its established contribution to reward learning.

To investigate whether dopamine modulates time-dependent coding of value, we administered the dopamine precursor L-dopa, the dopamine antagonist haloperidol, and placebo to healthy volunteers performing an intertemporal choice task. The task required subjects to make genuine choices between differing amounts of money, offered over variable time periods, mostly involving the choice between smaller-sooner versus larger-later monetary rewards. Such choices are well characterized by models that incorporate both the discounting effects of time and the discounting effects of increasing reward magnitude (diminishing marginal utility) (Pine et al., 2009). Accordingly, the discounted utility or subjective value of a delayed reward is determined by the product of the discount factor (a number between zero and one) and the utility of the reward. If dopamine modulates an individual's choice in this task, it might reflect a change in either the discount rate or the utility concavity/convexity (see Materials and Methods)—a distinction that we were able to probe here at both behavioral and neurophysiological levels, using functional magnetic resonance imaging (fMRI). Additionally, we assessed whether dopamine had any 


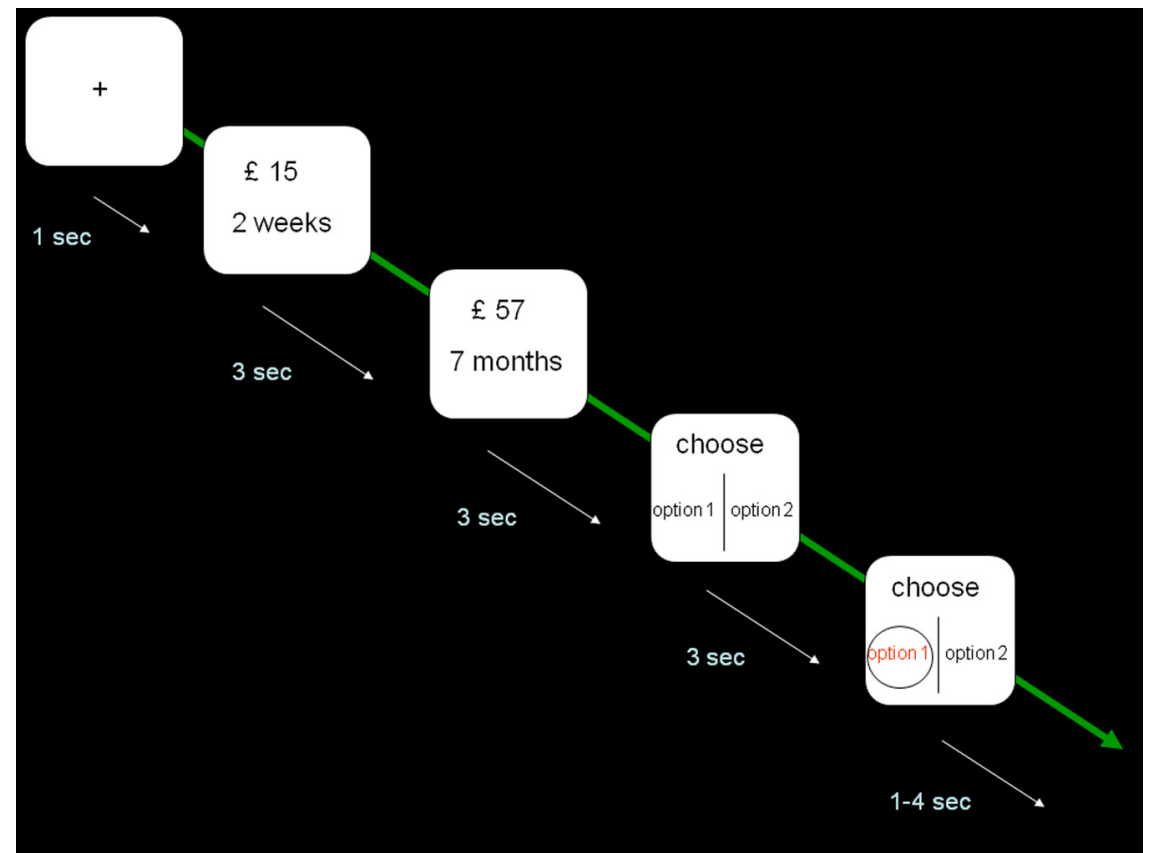

Figure 1. Task design. Subjects were given a set of 220 intertemporal binary choices, mostly between smaller-sooner and larger-later rewards, that varied in amounts from $£ 1-£ 150$ with associated delays of $1-52$ weeks. Note that option presentation and choice phases were presented serially to derive less ambiguous fMRI data, uncompounded by factors such as comparative valuation. Subjects were given the same array of choices to make under each of the three drug conditions. One trial was chosen at random at the end of each testing session and the chosen option was paid via a bank transfer at the specified delay.

effect on the rate of slowing down engendered by decisionconflict (Frank et al., 2007; Pochon et al., 2008) to distinguish global from discrete influences on impulsivity.

\section{Materials and Methods}

We used fMRI while subjects chose between two serially presented options of differing magnitude (from $\mathfrak{£} 1$ to $£ 150$ ) and delay (from 1 week to 1 year) (Fig. 1). Each subject performed the task on three separate occasions (relating to the three drug conditions). These choices were often smaller-sooner versus larger-later options. One of the subjects' choices was selected at random at the end of the experiment (in each experimental session) and paid for real (i.e., at the specified future date) by bank transfer. We used subjects' choices to assess the extent of discounting for both magnitude and time. We assessed a model that combined a utility function (converting magnitude to utility) with a standard hyperbolic discounting function. In simple terms, the function for the discounted utility (subjective value) of a delayed reward $(V)$ is equal to $D \times U$ where $D$ is a discount factor between 0 and 1 and $U$ is undiscounted utility. $D$ is typically a hyperbolic function of the delay to the reward and incorporates the discount rate parameter $(K)$, which determines how quickly one devalues future rewards. $U$ is (typically) a concave function of the magnitude of a reward and depends on an individual parameter $(r)$ that determines the concavity/convexity of the function, or the rate of diminishing marginal utility for gains and consequently the instantaneous value of the larger relative to the smaller reward. The greater $K$ or $r$, the more the individual is likely to choose the sooner option and therefore the more impulsive is the individual (Ho et al., 1999; Pine et al., 2009). In accordance with utility theory, choice is determined by the principle of utility maximization whereby the option with the greatest discounted utility is selected.

Participants. Fourteen right-handed, healthy volunteers were included in the experiment ( 6 males; 8 females; mean age, 21; range, 18-30). Subjects were preassessed to exclude those with a prior history of neurological or psychiatric illness. All subjects gave informed consent and the study was approved by the University College London ethics committee. One subject dropped out of the study after the first session and was not included in the results. Another did not complete the final (placebo) session in the scanner, but their behavioral data from all sessions and imaging data from two sessions were included in the results.

Procedure and task description. Each subject was tested on three separate occasions. Upon arrival on each occasion, subjects were given an instruction sheet to read explaining how the drug blinding would be implemented. They then completed a visual analog scale (Bond and Lader, 1974) that measured subjective states such as alertness, and were subsequently given an envelope containing two pills that were either $1.5 \mathrm{mg}$ of haloperidol or placebo. One and a half hours after taking the first set of pills, subjects were given another envelope containing two pills that were either Madopar (containing $150 \mathrm{mg}$ of L-dopa) or placebo. The placebo tablets (vitamin $\mathrm{C}$ or multivitamins) were indistinguishable from the drugs. In all, each subject received one dose of Madopar on one session, one dose of haloperidol on another, and on one session both sets of tablets were placebo. The order of each drug condition in relation to the testing session was counterbalanced across subjects and was unknown to the experimenter to achieve a double-blind design. Testing commenced $30 \mathrm{~min}$ after ingestion of the second set of tablets. The timings were aimed to achieve a peak plasma concentration of the drug approximately halfway through the testing. After testing, subjects completed another (identical) visual analog scale. No two testing sessions occurred within 1 week of each other.

The behavioral task was mostly as described by Pine et al. (2009). Each trial consisted of a choice between a smaller-sooner reward and a largerlater reward. The choice was presented serially, in three stages (Fig. 1). The first two stages consisted of presentation of the details of each option, i.e., the magnitude of the reward in pounds and the delay to its receipt in months and weeks. After presentation of the options, a third screen prompted the subject to choose between option 1 (the option presented first) or option 2, by means of a button-box, using their right hand. A $3 \mathrm{~s}$ delay followed each of the three phases. The choice could only be made during the $3 \mathrm{~s}$ following presentation of the choice screen. Once a choice had been made, the chosen option was highlighted in blue. Providing there was sufficient time, the subject could change his/her mind. There was a jittered delay of 1-4 s following the choice phase, followed by presentation of a fixation cross for $1 \mathrm{~s}$.

The experiment consisted of a total of 200 trials. Option 1 was the smaller-sooner reward in $50 \%$ of trials. In addition, we included a further 20 "catch" trials, where one of the options was both greater in value and available sooner than the other one. These catch trials occurred approximately every tenth trial and enabled us to ascertain how well the subjects were concentrating on the task, under the assumption that the norm was to prefer the larger-sooner reward in these choices. Each subject was given the same array of choices on each testing session (i.e., each drug condition) with the exception of the first two subjects who were given a different set of choices on their first testing session. The option values were created using randomly generated magnitudes varying from $£ 1$ to $£ 150$ in units of $£ 1$ and delays ranging from 1 week to 1 year in units of single weeks (but presented as a number of months and weeks), again with a random distribution. This random nature of the values helped in orthogonalising magnitude and delay. To create choices between smaller-sooner and largerlater rewards, we introduced the constraint that the option with greater magnitude should be delayed more than the smaller, and vice versa for the catch trials. Subjects were assigned to one of two choice arrays depending on their responses within practice trials in their first session. This was done to match the presented choices to the level of impulsivity of the subject.

Payment was performed using a lottery to select one trial from each testing session. To impose ecological validity, we used a payment system 
that ensured that all the choices would be made in a realistic manner, with realistic consequences. Crucial to this design was the random selection of one of the choices made during the experiment, with real payment of the option chosen for that choice. This was achieved by way of a bank transfer made at the time associated with, and consisting of the amount of the selected option. Payment selection was implemented using a manual lottery after completion of all testing. The lottery contained 220 numbered balls, each representing a single trial from the task. The ball that was selected corresponded to the rewarded trial for that testing session. The magnitude and delay of the option that the subject chose in the selected trial was determined and awarded using a bank transfer. Thus, the payment each subject received was determined by a combination of the lottery and the choices that they made - a manipulation that ensured subjects treated all choices as real. The payment system was designed so that on average each subject would receive $\mathfrak{E} 75$ per session. No other payment was awarded for participation in the experiment.

Before subjects were taken into the scanner, they were shown the lottery machine and given an explanation as to how the bank transfer would be implemented, to reassure them that the payment and selection system was genuine. After a short practice of six trials, they were taken into the scanner where they performed two sessions of 110 trials each, lasting in total $\sim 50 \mathrm{~min}$

Imaging procedure. Functional imaging was conducted by using a 3-tesla Siemens Allegra head-only MRI scanner to acquire gradient echo T2*-weighted echo-planar images (EPI) with blood oxygenation leveldependent (BOLD) contrast. We used a sequence designed to optimize functional sensitivity in the orbitofrontal cortex (Deichmann et al., 2003). This consisted of tilted acquisition in an oblique orientation at $30^{\circ}$ to the anterior cingulate-posterior cingulate AC-PC line, as well as application of a preparation pulse with a duration of $1 \mathrm{~ms}$ and amplitude of $-2 \mathrm{mT} / \mathrm{m}$ in the slice selection direction. The sequence enabled 36 axial slices of $3 \mathrm{~mm}$ thickness and $3 \mathrm{~mm}$ in-plane resolution to be acquired with a repetition time (TR) of $2.34 \mathrm{~s}$. Subjects were placed in a light head restraint within the scanner to limit head movement during acquisition. Functional imaging data were acquired in two separate 610 volume sessions. A T1-weighted structural image and fieldmaps were also acquired for each subject after the testing sessions.

Behavioral analysis. To obtain an overall measure of impulsive choice, we counted the number of sooner options chosen out of the 220 trials, under each drug condition, for each subject. Trials where a response was not made were excluded from this sum in all three drug conditions. For example, if one subject did not respond in time for trial number 35 in the placebo condition, this trial was excluded from the count in the other two conditions for that subject. This ensured that the comparisons were made on a trial-by-trial basis (as the same array of trials was given in each testing session) and any effect of drug on this measure was not related to the number of choices made in each condition. A repeated-measures ANOVA was used to look for any differences in this overall measure across drug conditions.

Parameter estimation. We implemented the softmax decision rule to assign a probability $\left(P_{\mathrm{O} 1}\right.$ for option 1$)$ to each option of the choice given the value of the option ( $V_{\mathrm{O} 1}$ for option 1$)$ whereby

$$
P_{\mathrm{O} i}=\frac{e^{\left(V_{\mathrm{O} i} / \beta\right)}}{e^{\left(V_{01} / \beta\right)}+e^{\left(V_{02} / \beta\right)}} .
$$

$V_{\mathrm{O} i}$ represents the value of an option (i.e., a delayed reward) according to a particular model of option valuation (see below). The $\beta$ parameter represents the degree of stochasticity of the subject's behavior (i.e., sensitivity to the value of each option).

We used a discounted utility model of option valuation, which we previously reported (Pine et al., 2009) as providing an accurate fit to subject's choices in this task. This model states that the discounted utility $(V)$ of a reward of magnitude $(M)$ and with a delay $(d)$ can be expressed as follows:

$$
V=D(d) \cdot U(M)=\frac{1-e^{(-r \cdot M)}}{r(1+K \cdot d)},
$$

where

$$
D=\frac{1}{1+K \cdot d}
$$

and

$$
U=\frac{1-e^{(-r \cdot M)}}{r}
$$

$D$ can be thought of as the discount factor-the delay-dependent factor (between 0 and 1 ) by which the utility is discounted in a standard hyperbolic fashion (Mazur, 1987). The discount rate parameter $K$ quantifies an individual's tendency to discount the future such that a person with a high $K$ quickly devalues rewards as they become more distant. $U$ is undiscounted utility and is governed by the magnitude of each option and $r$, a free parameter governing the curvature of the relationship. The greater the value of $r$, the more concave the utility function, and where $r$ is negative, the utility function is convex. The greater $r$ (above zero), the greater the rate of diminishing marginal utility and the more impulsive is the individual in choice. Note that according to traditional models of intertemporal choice valuation, which do not take into account the discounting of magnitude (Mazur, 1987), impulsivity, defined by the propensity to choose the smaller-sooner option, is solely a function of $K$ and so the two might be expected to correlate perfectly. Hence, $K$ is often considered a measure of this trait. However, since the discounting of magnitude has also been shown to determine choice outcome in animals and humans (Ho et al., 1999; Pine et al., 2009), we prefer to equate impulsivity with choice behavior as the temporal discount rate does not perfectly correlate with this key measure.

To calculate the maximum likelihood parameters for each model as well as a measure of the fit, maximum likelihood estimation was used. Each of the parameters (including $\beta$ ) was allowed to vary freely. For each subject, the probability was calculated for each of the 220 options chosen from the 220 choices (including catch trials), using the softmax formula and implemented with optimization functions in Matlab (MathWorks). The log-likelihood was calculated using the probability of the option chosen at trial $t\left(P_{\mathrm{O}(t)}\right)$ from Eq. 1 such that

$$
\ln L=\sum_{t} \ln P_{\mathrm{O}(t)} \text {. }
$$

A repeated-measures ANOVA was used to test for any differences in the discount rate $(K)$ and the utility concavity $(r)$ across drug conditions.

For the purposes of the imaging and reaction time analyses, a further estimation was performed whereby all the choices from each subject in each condition were grouped together (as if made by one subject) and modeled as a canonical subject to estimate canonical parameter values (using the fitting procedure above, Parameter estimation). This was performed to reduce the noise associated with the fitting procedure at the single-subject level. In addition, we did not wish to build the behavioral differences into our regression models when analyzing the fMRI data, as we sought independent evidence for our behavioral findings.

Imaging analysis. Image analysis was performed using SPM5 (www. fil.ion.ucl.ac.uk/spm). For each session, the first five images were discarded to account for $\mathrm{T} 1$ equilibration effects. The remaining images were realigned to the sixth volume (to correct for head movements), unwarped using fieldmaps, spatially normalized to the Montreal Neurological Institute (MNI) standard brain template, and smoothed spatially with a three-dimensional Gaussian kernel of $8 \mathrm{~mm}$ full-width at halfmaximum (FWHM) (and resampled, resulting in $3 \times 3 \times 3 \mathrm{~mm}$ voxels). Low-frequency artifacts were removed using a $1 / 128 \mathrm{~Hz}$ high-pass filter and temporal autocorrelation intrinsic to the fMRI time series was corrected by prewhitening using an $\mathrm{AR}(1)$ process.

Single-subject contrast maps were generated using parametric modulation in the context of the general linear model. We performed an analysis, examining variance in regional BOLD response attributable to different regressors of interest: $U, D$, and $V$ for all options over all drug conditions. This allowed us to identify regions implicated in the evaluation and integration of different components of value (in the placebo 
Table 1. Summary of behavioral findings

\begin{tabular}{|c|c|c|c|c|c|c|c|c|c|}
\hline \multirow[b]{2}{*}{ Subject } & \multicolumn{3}{|l|}{ Placebo } & \multicolumn{3}{|l|}{ L-Dopa } & \multicolumn{3}{|l|}{ Haloperidol } \\
\hline & Catch trials & No. of sooner chosen & $K$ value & Catch trials & No. of sooner chosen & $K$ value & Catch trials & No. of sooner chosen & $K$ value \\
\hline 1 & 20 & & 0.0628 & 20 & & 0.0632 & 20 & & 0.0225 \\
\hline 2 & 20 & & 0.0419 & 19 & & 0.0831 & 19 & & 0.0039 \\
\hline 3 & 20 & 119 & 0.0277 & 20 & 126 & 0.037 & 19 & 133 & 0.0364 \\
\hline 4 & 20 & 147 & 0.0209 & 20 & 151 & 0.0276 & 20 & 119 & 0.0175 \\
\hline 5 & 20 & 69 & 0.0048 & 20 & 163 & 0.0581 & 20 & 59 & 0.0036 \\
\hline 6 & 20 & 34 & 0.0029 & 20 & 46 & 0.0052 & 20 & 43 & 0.0023 \\
\hline 7 & 20 & 139 & 0.0287 & 20 & 196 & 0.1122 & 20 & 174 & 0.0679 \\
\hline 8 & 19 & 109 & 0.0214 & 20 & 148 & 0.0384 & 20 & 160 & 0.0452 \\
\hline 9 & 18 & 159 & 0.0289 & 20 & 165 & 0.0447 & 18 & 170 & 0.1298 \\
\hline 10 & 20 & 178 & 0.0596 & 20 & 186 & 0.0723 & 20 & 174 & 0.0758 \\
\hline 11 & 20 & 32 & 0.0028 & 20 & 51 & 0.0052 & 20 & 43 & 0.0024 \\
\hline 12 & 20 & 55 & 0.0053 & 20 & 89 & 0.0093 & 20 & 31 & 0.0014 \\
\hline 13 & 20 & 165 & 0.0003 & 20 & 170 & 0.0239 & 19 & 165 & 0.0477 \\
\hline Mean & 19.769 & 109.636 & 0.024 & 19.923 & 135.545 & 0.045 & 19.615 & 115.545 & 0.035 \\
\hline
\end{tabular}

Subjects more often chose the smaller-sooner reward in L-dopa versus placebo conditions (note subjects 1 and 2 performed different choice arrays across conditions so cannot be meaningfully compared in this way). Discount rate paramete (K) was greater under L-dopa than placebo conditions. Subjects nearly always answered catch trials (out of 20) correctly, i.e., choosing the larger-sooner option. (An expanded set of results, including estimates of the $r$ and $\beta$ parameters as well as the model fit scores, can be found in supplemental Table 1, available at www.jneurosci.org as supplemental material.)

condition) and to look for any differences in these activations across drug conditions.

$U, D$, and $V$ for each option (two per trial) were calculated using the canonical parameter estimates ( $K$ and $r$ ) in the context of our discounted utility model and convolved with the canonical hemodynamic response function (HRF) at the onset of each option. All onsets were modeled as stick functions and all regressors in the same model were orthogonalized (in the orders stated above) before analysis by SPM5. To correct for motion artifacts, the six realignment parameters were modeled as regressors of no interest in each analysis. In an additional analysis, we removed any potential confound relating to the orthogonalization of the regressors in our fMRI analysis by implementing another regression model but now removing the orthogonalization step. Here regressors were allowed to compete for variance such that in this more conservative model any shared variance components were removed, revealing only unique components of $U, D$, and $V$. Under this model, we again observed the same differences in $D$ and $V$ across drug conditions and no difference in $U$, although the magnitude of the differences was reduced.

At the second level (group analysis), regions showing significant modulation by each of the regressors specified at the first level were identified through random-effects analysis of the $\beta$ images from the single-subject contrast maps. We included the change in impulsivity measure (difference in number of sooner chosen) as a covariate when performing the contrast relating to differences in L-dopa and placebo trials. We report results for regions where the peak voxel-level $t$ value corresponded to $p<0.005$ (uncorrected), with minimum cluster size of five. Coordinates were transformed from the MNI array to the stereotaxic array of Talairach and Tournoux (1988) (http://imaging.mrc-cbu. cam.ac.uk/imaging/MniTalairach).

The structural T1 images were coregistered to the mean functional EPI images for each subject and normalized using the parameters derived from the EPI images. Anatomical localization was performed by overlaying the $t$ maps on a normalized structural image averaged across subjects and with reference to the anatomical atlas of Mai et al. (2003).

Decision latency data. To examine the effect of decision conflict (choice difficulty) on decision latency, we calculated a measure of difficulty for each of the 220 choices by calculating the difference in discounted utility $(\Delta V)$ of the two options. This measure was calculated using the discounted utility model and the canonical parameter estimates (for the same reason they were used in the fMRI analyses). A linear regression was then performed to model the relationship between the decision latency for each choice and the difficulty measure. The parameter estimates ( $\beta \mathrm{s})$ were then used as a summary statistic and a second level analysis was performed by means of a one-sample $t$ test comparing the $\beta$ s against zero. This was performed separately for the group in each drug condition. To test for any differences in the relationship between conflict and latency across drug conditions, we used paired samples $t$ tests.

\section{Results}

We first analyzed the effects of the drug manipulation on behavior by considering the proportion of smaller-sooner relative to larger-later options chosen, of a total of 220 choices, made in each condition. These data revealed a marked increase in the number of sooner options chosen in the L-dopa condition relative to the placebo condition (mean 136 vs $110, p=0.013$ ) (Table 1, Fig. 2). Strikingly, this pattern was observed in all subjects where this comparison could be made. There was no significant difference between haloperidol and placebo conditions on this disposition. Note, the task consisted of the same choice array in each condition.

We next used maximum likelihood estimation to find the best-fitting parameters ( $K$ and $r$ ) for the discounted utility model, for each subject in each condition, to determine whether a specific effect on either of these parameters mediated the observed increase in behavioral impulsivity. By comparing the estimated parameters controlling the discount rate and utility concavity across conditions, a specific effect of $\mathrm{L}$-dopa on the discount rate was found, with no effect on utility concavity (Table 1, Fig. 2, and supplemental Table 1, available at www.jneurosci.org as supplemental material). Thus, under L-dopa, a higher discount rate was observed relative to placebo ( $p=0.01$ ), leading to a greater devaluation of future rewards. By way of illustration, using a group canonical parameter estimate to plot a discount function for each drug condition, it can be seen that under placebo it required a delay of $\sim 35$ weeks for a $£ 150$ reward to have a present (subjective) value of $\mathfrak{E} 100$, however, under L-dopa the same devaluation took place with a delay of just 15 weeks (Fig. 2). Canonical parameter estimates used for the imaging analyses were 0.0293 for $K$ and 0.0019 for $r$ (all values of $K$ reported are calculated from time units of weeks).

In accordance with Pine et al. (2009), parameter estimates for each subject (across conditions) were greater than zero, revealing both a significant effect of temporal discounting $(p<0.001)$ and nonlinearity (concavity) of instantaneous utility $(p<0.05)$. Note that unlike traditional models of intertemporal choice (Mazur, 1987 ), where choice outcome is solely a function of $K$, the model used here entails that the number of sooner options chosen also depends on the $r$ parameter (see Materials and Methods) (Pine et al., 2009) and hence $K$ is not in itself a pure measure of choice impulsivity. Further, the accuracy of estimated parameters de- 


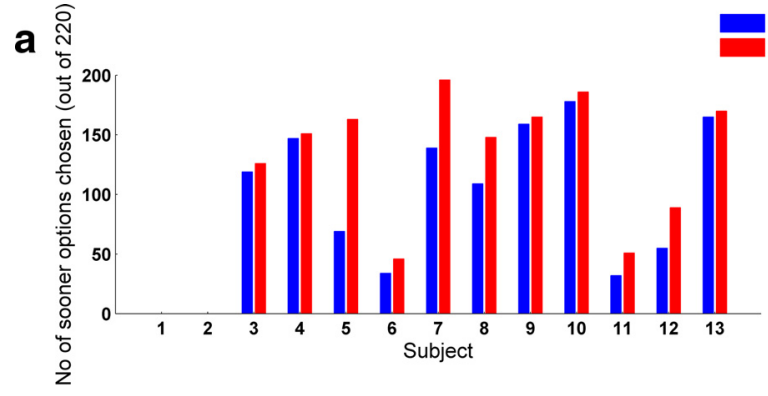

b

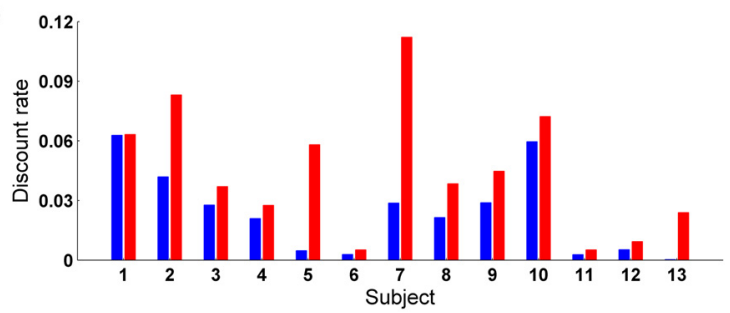

$\mathbf{C}$

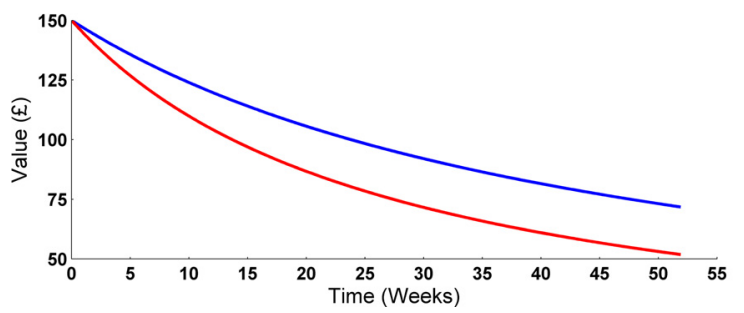

Figure 2. Behavioral comparisons and parameter estimates in placebo and L-dopa conditions. $\boldsymbol{a}$, Subjects performed exactly the same set of (220) choices under all three treatment conditions but more often chose the smaller-sooner than larger-later option after taking L-dopa. For clarity of presentation, data for haloperidol is not shown, as these data did not differ from placebo. (Note that subjects 1 and 2 performed a different set of choices under each condition and so cannot be compared in this way). $\boldsymbol{b}$, Maximum likelihood estimation of the individual parameters revealed that subjects had a higher discount rate under L-dopa than placebo. $c$, The estimated discount function for a $£ 150$ reward over the course of a 52 week delay, using the group parameter estimate, reveals a much steeper devaluation of future rewards under L-dopa relative to placebo.

pends on both the stochasticity and consistency of subjects' responses. For example, the estimated parameters in subject 13's placebo trial were anomalous in relation to the rest of the data (supplemental Table 1, available at www.jneurosci.org as supplemental material), indicating this subject could have made inconsistent choices in this session. When comparing across subjects, note that the number of sooner choices made is also dependent on the choice set the subject received (one of two).

Additionally, we examined whether a slowing down in decision latencies was apparent as choices became increasingly difficultconsequent upon increasing closeness in option values-and whether any group differences were apparent on this measure. We performed a regression to assess the relationship between decision latency and the difficulty of each choice as measured by the difference in discounted utility $(\Delta V)$ between the two choice options, calculated using the estimated parameter values. In placebo $(p<$ $0.001)$, L-dopa $(p<0.001)$, and haloperidol $(p<0.001)$ conditions, subjects' decision latencies increased as $\Delta V$ got smaller, that is, as the difference in subjective value between the options got smaller. However, no overall difference was observed in this measure across drug conditions. This indicates that, unlike the choice outcome, dopamine manipulation did not influence the amount of time given to weigh a decision, or ability to "hold your horses," and corroborates the suggestion that impulsivity is not a unitary construct (Evenden,
1999; Ho et al., 1999; Winstanley et al., 2004a; Dalley et al., 2008). This observation accords with a previous finding that dopamine medication status in PD was not associated with change in decision latencies in a different choice task (Frank et al., 2007).

Subjective effects were analyzed by comparing changes in the three factors identified by Bond and Lader (1974), namely, alertness, contentedness, and calmness, relative to the change in scores observed in the placebo condition. Differences were found in the haloperidol versus placebo conditions, where subjects were less alert under haloperidol $(p<0.05)$.

To establish how enhanced impulsivity under L-dopa was represented at a neural level, we applied three (orthogonalized) parametric regressors, $U, D$, and $V$, associated with the presentation of each option, as dictated by our model, to the brain imaging data. The regressors were created for each subject, in each condition, using canonical parameter values estimated from all subjects' choices over all sessions, in a test of the null hypothesis that brain activity does not differ between conditions.

In a preliminary analysis, we examined correlations for these three regressors in the placebo condition to replicate previous findings (Pine et al., 2009). Our results (supplemental Results, available at www.jneurosci.org as supplemental material) were consistent with those shown previously, in that $D, U$, and $V$ all independently correlated with activity in the caudate nucleus (among other regions). This supports a hierarchical, integrated view of option valuation where subcomponents of value are dissociably encoded and then combined to furnish an overall value used to guide choice.

The critical fMRI analyses focused on the key behavioral difference in option valuation under L-dopa compared with placebo conditions. When comparing neural activity for $U, D$, and $V$, significant differences were found for both $D$ and $V$, a finding that matches the behavioral result. Specifically, we observed enhanced activity in regions relating to the discount factor $D$ under L-dopa relative to placebo conditions (Fig. $3 a$ and supplemental Results, available at www.jneurosci.org as supplemental material) and no effect of haloperidol (that is, the regression coefficients in the placebo and haloperidol condition did not differ significantly). These regions included the striatum, insula, subgenual cingulate, and lateral orbitofrontal cortices. These results show that the characteristic decrease in activity of these regions as rewards become more delayed (or increase as they become temporally closer) (McClure et al., 2004; Tanaka et al., 2004; Kable and Glimcher, 2007; Pine et al., 2009) (see also supplemental Results for placebo, available at www.jneurosci.org as supplemental material) is more marked in the L-dopa relative to placebo conditions, in a manner that parallels the behavioral finding, where L-dopa increased preference for sooner rewards by increasing the discount rate, thereby rendering sooner rewards more attractive relative to later rewards. Moreover, just as there was no significant difference in the estimated $r$ parameter across these trials, we observed no significant difference in $U$ activity between L-dopa and placebo trials, indicating that L-dopa did not affect the encoding of reward utility.

Previous studies (Kable and Glimcher, 2007; Pine et al., 2009), as well as an analysis of the placebo group alone, implicate striatal regions, among others, in encoding discounted utility $(V)$. When comparing regions correlating with $V$, decreased activity was observed in caudate, insula, and lateral inferior frontal regions, in L-dopa compared with placebo conditions (Fig. $3 b$ and supplemental Results, available at www.jneurosci.org as supplemental material). This result indicates that for a reward of a given magnitude and delay, reduced activity in regions encoding subjective 


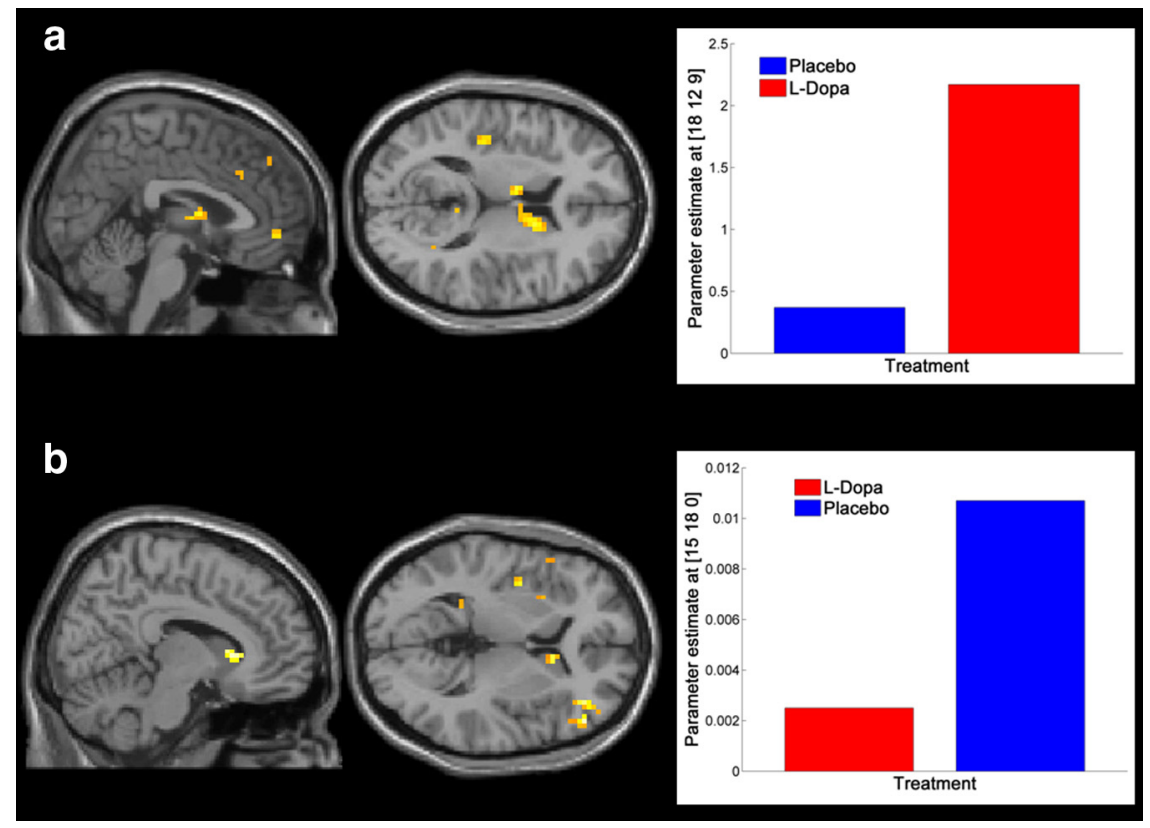

Figure 3. Differences in neural activity between L-dopa and placebo conditions in response to subjective value and the discount factor (statistical parametric maps and parameter estimates). $\boldsymbol{a}$, Regions that correlated with the discount factor (D) (i.e., reward proximity) and were significantly more active in L-dopa compared with placebo trials. $\boldsymbol{b}$, Regions that correlated with the discounted utility ( $V$ ) or subjective value of the options and were significantly more active in placebo relative to L-dopa trials. Bar charts indicate the mean parameter estimates at the peak striatal voxel relating to $D$ (in $\boldsymbol{a}$ ) and $V$ (in $\boldsymbol{b}$ ) activity. (See supplemental Results for extensive results, available at www.jneurosci.org as supplemental material).

value (discounted utility) was engendered by L-dopa. This reduction was associated with the enhanced temporal discounting, and led to an increase in the selection of smaller-sooner (impulsive) options in this condition relative to placebo.

Because the fMRI data used the same single set of canonical parameters (across all conditions, testing the null hypothesis that they are all the same), these findings accord with the behavioral results whereby increasing the discount rate under L-dopa leads to a reduction in $D$, leading to a corresponding reduction in $V$ and, hence, an increased relative preference for sooner rewards. Note that if dopamine encoded discounted utility alone, one would predict the opposite result, with greater activity in the L-dopa condition.

Inspection of the behavioral results (Table 1, Fig. 2) revealed that an increase in impulsivity following L-dopa was expressed to a greater extent in some subjects than in others. On this basis, we performed a covariate analysis on the previous contrasts by calculating a difference score of the number of sooner options chosen in the placebo and L-dopa trials. The larger this metric, the greater the increase in impulsivity (discount rate) induced by $\mathrm{L}$-dopa. By regressing this quantity as a covariate in the contrast comparing $D$ in L-dopa minus placebo conditions (Fig. $3 a$ ), we found a significant correlation with activity in the amygdala (bilaterally) (Fig. 4). Because the difference in choice score across subjects may have been partially affected by the fact that subjects were assigned to one of two possible choice sets, and to increase power (being able to include more subjects), we repeated this analysis, this time using the difference in estimated $K$ values from placebo to L-dopa trials. The result of this analysis (see supplemental Results, available at www.jneurosci.org as supplemental material) again demonstrated a strong positive correlation between amygdala activity and degree of increase in $K$ from placebo to L-dopa trials. These results suggest that individual subject susceptibility to impulsivity under the influence of L-dopa is modu- lated by the degree of amygdala response to temporal proximity of reward.

\section{Discussion}

Existing theories of dopamine focus on its role in reward learning, where dopamine is thought to mediate a prediction error signal used to update the values of states and actions that allow prediction and control, respectively, during decisionmaking. These models have been used to illustrate how abnormal dopamine processing might lead to impulsive and addictive behaviors, on the basis of experience (i.e., through learning) (Redish, 2004; Frank et al., 2007; Dagher and Robbins, 2009). Here, a distinct aspect of impulsivity was explicitly probed, based on the relationship of the timing of rewards and their utility, independently of feedback and learning. In intertemporal choice, decision-makers must choose between rewards of differing magnitude and delay. This is achieved by discounting the value of future amounts of utility (in accordance with their delay) to compare their present values. Within this framework, dopamine could potentially increase impulsive choice in two distinct ways (Pine et al., 2009), as follows: as a result of an increased rate of diminishing marginal utility for gains (which would decrease the subjective instantaneous value of larger magnitude relative to smaller magnitude rewards), or through enhanced temporal discounting of future rewards. Our results suggest that dopamine selectively impacts on the discount rate, without any significant effect on the utility function. Moreover, these behavioral results were independently supported by the fMRI data in that the key difference engendered by L-dopa was a modulation of neural responses in regions associated with the discounting of rewards and, consequently, their overall subjective value, with no effects evident for the actual utility of rewards. In summary, this study provides evidence that dopamine controls how the timing of a reward is incorporated into the construction of its ultimate value. This suggests a novel mechanism through which dopamine controls human choice and, correspondingly, traits such as impulsiveness.

Our results add weight to the suggestion that impulsivity is not a unitary construct and moreover that different subtypes of impulsiveness can be dissociated pharmacologically and neurobiologically (Evenden, 1999; Ho et al., 1999; Winstanley et al., 2004a; Dalley et al., 2008). The effects of dopamine were only observable in impulsive choice as measured by choice outcome/preference but did not impact on deliberation- "holding your horses" (Frank et al., 2007) — that occurs when options are closely valued, engendering decision-conflict (Botvinick, 2007; Pochon et al., 2008) also related to reflection or preparation impulsiveness (Evenden, 1999; Clark et al., 2006).

No human study has as yet demonstrated dopamine's propensity to enhance temporal impulsiveness. Previous dopamine manipulations in rodents have shown inconsistent effects in intertemporal choice, with some showing that dopamine enhancement leads to a decrease in impulsive choice or that dopamine attenuation leads to an increase (Richards et al., 1999; Cardinal et 

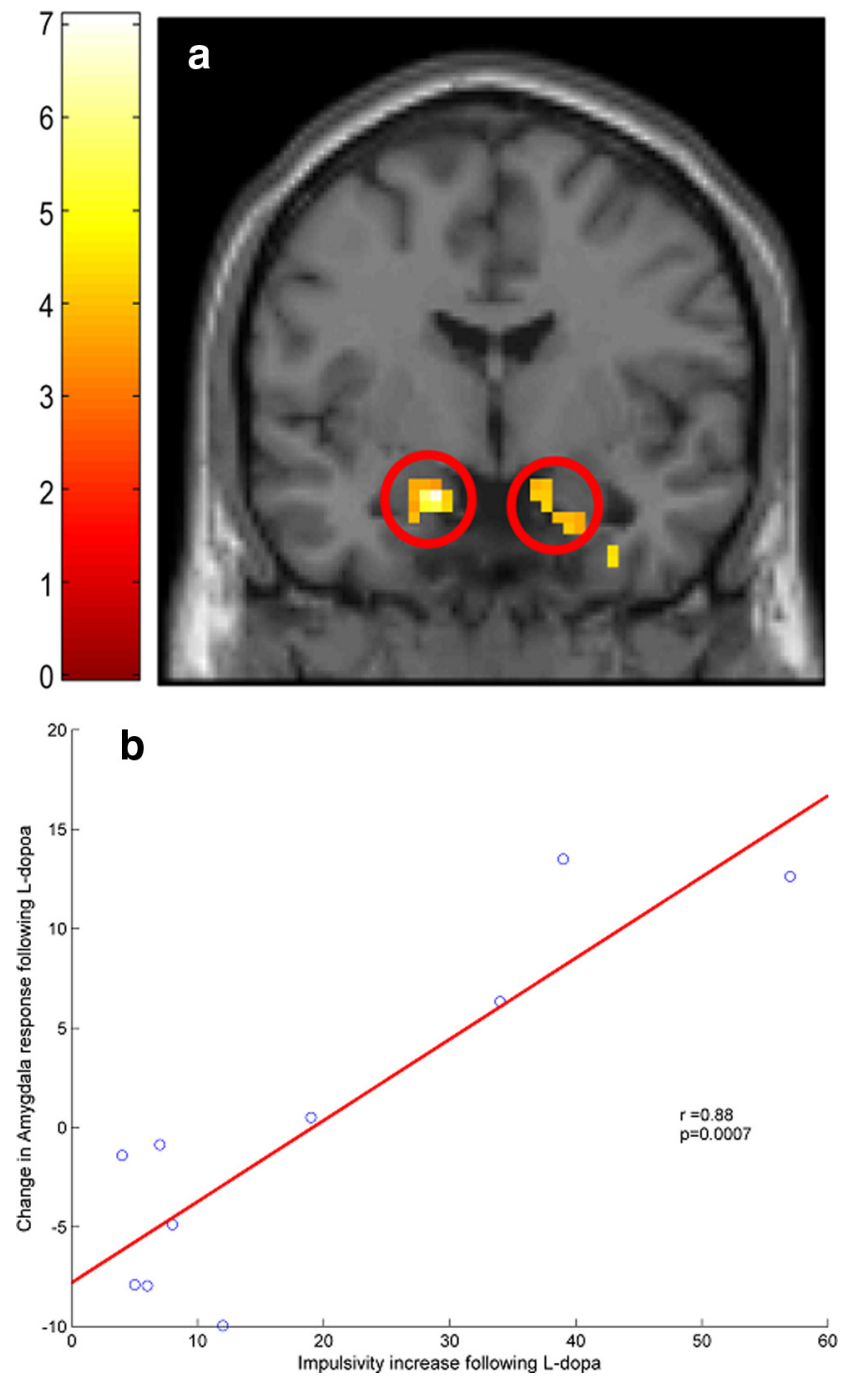

Figure 4. Intersubject variability in increase in impulsivity following L-dopa. $\boldsymbol{a}$, Statistical parametric map showing areas expressing an overall sensitivity to the discount factor (in L-dopa minus placebo conditions) and that covaried with the degree to which choices became more impulsive following L-dopa, relative to placebo, on a subject-by-subject basis. A significant correlation was observed bilaterally in the amygdala (supplemental Results, available at www. jneurosci.org as supplemental material). $\boldsymbol{b}$, Change in BOLD response in the amygdala as reward proximity increased, in relation to the degree to which each subject became more impulsive under L-dopa.

al., 2000; Wade et al., 2000; Isles et al., 2003; Winstanley et al., 2003; van Gaalen et al., 2006; Bizot et al., 2007; Floresco et al., 2008), whereas others demonstrate the opposite, a dosedependent effect, or no effect (Logue et al., 1992; Charrier and Thiébot, 1996; Evenden and Ryan, 1996; Richards et al., 1999; Cardinal et al., 2000; Isles et al., 2003; Helms et al., 2006; Bizot et al., 2007; Floresco et al., 2008). A number of factors may contribute to these discrepancies, namely, whether the manipulation occurs prelearning or postlearning, whether a cue is present during the delay, presynaptic versus postsynaptic drug effects, the paradigm used, the drug used/receptor targeted, the involvement of serotonin, and particularly the drug dosage. Human studies of intertemporal choice have observed an increase in self-control (de Wit et al., 2002) or no effect (Acheson and de Wit, 2008; Hamidovic et al., 2008) when enhancing dopamine function. Most of these studies are complicated by their use of monoaminergic stimulants such as amphetamine or methylphenidate, which are often thought to decrease impulsivity. These studies could be confounded by the concomitant release of serotonin (Kuczenski and Segal, 1997), which is also implicated in the modulation of intertemporal choice. Specifically, it has been shown that enhancing serotonin function can reduce impulsiveness in intertemporal choice or vice versa (Wogar et al., 1993; Richards and Seiden, 1995; Poulos et al., 1996; Ho et al., 1999; Mobini et al., 2000) and that destruction of serotonergic neurons can block the effects of amphetamine (Winstanley et al., 2003). Furthermore, it is thought that, on the basis of extensive evidence, moderate doses of amphetamine reduce dopamine neurotransmission via presynaptic effects, which may explain its dose-dependent effects in many previous studies as well as its therapeutic efficacy (in moderate doses) in a putatively hyperdopaminergic ADHD (Seeman and Madras, 1998, 2002; Solanto, 1998, 2002; Solanto et al., 2001; de Wit et al., 2002). L-Dopa has not previously been used to affect impulsive choice, and perhaps offers more compelling and direct evidence for dopamine's role. Although L-dopa can lead to increases in noradrenaline and its precise mode of action is not well understood, noradrenaline is not thought to play a major role in the regulation of intertemporal choice (van Gaalen et al., 2006). Additionally, it is possible that L-dopa could have caused subjective effects that were not picked up by the subjective scales used here.

Our failure to find a corresponding reduction in impulsivity relative to placebo with administration of the putative dopaminergic antagonist haloperidol is likely to reflect a number of factors. These include haloperidol's nonspecific and widespread pharmacological effects or dosage-some studies indicate haloperidol may paradoxically boost dopamine in small doses, due to presynaptic effects on the D2 autoreceptor (Frank and O'Reilly, 2006). Additionally, the subjective effects caused by the drug, including reduction in alertness, may have made the data noisier. Further studies should use more specific dopamine antagonists to assess whether a reduction in dopamine function can decrease impulsivity in humans.

Dopamine is known to have a dominant effect on primitive reward behaviors such as approach and consummation (Parkinson et al., 2002). Such effects are consistent with a broad role in the construction of incentive salience (Berridge, 2007; Robinson and Berridge, 2008) and are more difficult to account for in terms of learning, per se. The mediation of unconditioned and conditioned responses by dopamine relates to the concept of Pavlovian impulsivity, where responses associated with primary, innate values form a simple, evolutionarily specified action set operating alongside, and sometimes in competition with, other control mechanisms, such as habit-based and goal-directed action (Dayan et al., 2006; Seymour et al., 2009). Importantly, these "Pavlovian values and actions" are characteristically dependent on spatial and temporal proximity to rewards and, as such, provide one possible mechanism via which dopamine could control the apparent rate of temporal discounting. If such a process underlay dopamine-induced impulsivity in this task, then it would suggest that this innate (Pavlovian) response system operates in a much broader context than currently appreciated, since the rewards in this task are secondary rewards occurring at a minimum of 1 week. This explanation stands in contrast to the idea of a selective dopaminergic enhancement of a system (based in limbic areas) that only values short-term rewards (McClure et al., 2004). Such a duel-system account would be difficult to reconcile with previous studies (Kable and Glimcher, 2007; Pine et al., 2009), which suggest that limbic areas value rewards at all delays. 
Such an account raises important questions about the amygdala-dependent susceptibility to dopamine-induced impulsivity that we observed in our data. Here, amygdala activity in response to $D$ covaried with the degree to which behavior became more impulsive following L-dopa. In Pavlovian-instrumental transfer (PIT), a phenomenon dependent on connectivity between amygdala and striatum (Cardinal et al., 2002; Seymour and Dolan, 2008), and whose expression is known to be modulated by dopamine (Dickinson et al., 2000; Lex and Hauber, 2008), appetitive Pavlovian values increase responding for rewards. Notably, individual susceptibility to this influence correlates with amygdala activity (Talmi et al., 2008), suggesting that the amygdala might modulate the extent to which primary conditioned and unconditioned reward values influence instrumental (habitbased and goal-directed) choice. If this is indeed the case, then it predicts that concurrent and independent presentation of reward-cues during intertemporal choice might elicit enhanced temporal impulsivity via an amygdala-dependent mechanism. We note evidence that basolateral amygdala lesions increase choice impulsivity in rodents (Winstanley et al., 2004b), an observation opposite to what we would expect based on the current data. In contrast, amygdala activity has previously been reported to correlate with the magnitude of temporal discounting in an fMRI study (Hoffman et al., 2008). These issues provide a basis for future research that can systematically test these divergent predictions in humans.

Lastly, these results speak to a wider clinical context and offer an explanation as to why an increase in impulsive and risky behaviors is observed in dopamine dysregulation syndrome, addiction, and ADHD, all of which are associated with hyperdopaminergic states caused by striatal dopamine flooding or sensitization (Solanto, 1998, 2002; Seeman and Madras, 2002; Berridge, 2007; Robinson and Berridge, 2008; Dagher and Robbins, 2009; O'Sullivan et al., 2009). In support of this thesis, Voon et al. (2009) found that dopamine medication status in PD patients with impulse-control disorders was associated with increased rates of temporal discounting. In conclusion, the results presented here demonstrate dopamine's ability to enhance impulsivity in humans and offer a novel insight into its role in modulating impulsive choice in the context of temporal discounting. These findings suggest that humans may be susceptible to temporary periods of increased impulsivity when factors that increase dopamine activity, such as the sensory qualities of rewards, are present during decision-making.

\section{References}

Acheson A, de Wit H (2008) Bupropion improves attention but does not affect impulsive behavior in healthy young adults. Exp Clin Psychopharmacol 16:113-123.

Ainslie G (1975) Specious reward: a behavioral theory of impulsiveness and impulse control. Psychol Bull 82: 463-496.

Berridge KC (2007) The debate over dopamine's role in reward: the case for incentive salience. Psychopharmacology 191:391-431.

Bickel WK, Marsch LA (2001) Toward a behavioral economic understanding of drug dependence: delay-discounting processes. Addiction 96:73-86.

Bickel WK, Miller ML, Yi R, Kowal BP, Lindquist DM, Pitcock JA (2007) Behavioral and neuroeconomics of drug addiction: competing neural systems and temporal discounting processes. Drug Alcohol Depend 90 [Suppl 1]:S85-S91.

Bizot JC, Chenault N, Houzé B, Herpin A, David S, Pothion S, Trovero F (2007) Methylphenidate reduces impulsive behaviour in juvenile Wistar rats, but not in adult Wistar, SHR and WKY rats. Psychopharmacology (Berl) 193:215-223.

Bond AJ, Lader MH (1974) The use of analogue scales in rating subjective feelings. Br J Med. Psychol 47:211-218.
Botvinick MM (2007) Conflict monitoring and decision making: reconciling two perspectives on anterior cingulate function. Cogn Affect Behav Neurosci 7:356-366.

Cardinal RN, Robbins TW, Everitt BJ (2000) The effects of d-amphetamine, chlordiazepoxide, alpha-flupenthixol and behavioural manipulations on choice of signalled and unsignalled delayed reinforcement in rats. Psychopharmacology 152:362-375.

Cardinal RN, Parkinson JA, Hall J, Everitt BJ (2002) Emotion and motivation: the role of the amygdala, ventral striatum, and prefrontal cortex. Neurosci Biobehav Rev 26:321-352.

Cardinal RN, Winstanley CA, Robbins TW, Everitt BJ (2004) Limbic corticostriatal systems and delayed reinforcement. Ann NY Acad Sci 1021:33-50.

Charrier D, Thiébot MH (1996) Effects of psychotropic drugs on rat responding in an operant paradigm involving choice between delayed reinforcers. Pharmacol Biochem Behav 54:149-157.

Clark L, Robbins TW, Ersche KD, Sahakian BJ (2006) Reflection impulsivity in current and former substance users. Biol Psychiatry 60:515-522.

Dagher A, Robbins TW (2009) Personality, addiction, dopamine: insights from Parkinson's disease. Neuron 61:502-510.

Dalley JW, Mar AC, Economidou D, Robbins TW (2008) Neurobehavioral mechanisms of impulsivity: fronto-striatal systems and functional neurochemistry. Pharmacol Biochem Behav 90:250-260.

Dayan P, Niv Y, Seymour B, Daw ND (2006) The misbehavior of value and the discipline of the will. Neural Netw 19:1153-1160.

Deichmann R, Gottfried JA, Hutton C, Turner R (2003) Optimized EPI for fMRI studies of the orbitofrontal cortex. Neuroimage 19:430-441.

de Wit H, Enggasser JL, Richards JB (2002) Acute administration of d-amphetamine decreases impulsivity in healthy volunteers. Neuropsychopharmacology 27:813-825.

Dickinson A, Smith J, Mirenowicz J (2000) Dissociation of Pavlovian and instrumental learning under dopamine agonists. Behav Neurosci 114:468-483.

Evenden JL (1999) Varieties of impulsivity. Psychopharmacology 146:348-361.

Evenden JL, Ryan CN (1996) The pharmacology of impulsive behaviour in rats: the effects of drugs on response choice with varying delays of reinforcement. Psychopharmacology 128:161-170.

Floresco SB, Tse MT, Ghods-Sharifi S (2008) Dopaminergic and glutamatergic regulation of effort- and delay-based decision making. Neuropsychopharmacology 33:1966-1979.

Frank MJ, O’Reilly RC (2006) A mechanistic account of striatal dopamine function in human cognition: psychopharmacological studies with cabergoline and haloperidol. Behav Neurosci 120:497-517.

Frank MJ, Samanta J, Moustafa AA, Sherman SJ (2007) Hold your horses: impulsivity, deep brain stimulation and medication in parkinsonism. Science 318:1309-1312.

Hamidovic A, Kang UJ, de Wit H (2008) Effects of low to moderate acute doses of pramipexole on impulsivity and cognition in healthy volunteers. J Clin Psychopharmacol 28:45-51.

Helms CM, Reeves JM, Mitchell SH (2006) Impact of strain and D-amphetamine on impulsivity (delay discounting) in inbred mice. Psychopharmacology (Berl) 188:144-151.

Ho MY, Mobini S, Chiang TJ, Bradshaw CM, Szabadi E (1999) Theory and method in the quantitative analysis of "impulsive choice" behaviour: implications for psychopharmacology. Psychopharmacology 146:362-372.

Hoffman WF, Schwartz DL, Huckans MS, McFarland BH, Meiri G, Stevens AA, Mitchell SH (2008) Cortical activation during delay discounting in abstinent methamphetamine dependent individuals. Psychopharmacology 201:183-193.

Isles AR, Humby T, Wilkinson LS (2003) Measuring impulsivity in mice using a novel operant delayed reinforcement task: effects of behavioural manipulations and d-amphetamine. Psychopharmacology (Berl) 170:376-382.

Kable JW, Glimcher PW (2007) The neural correlates of subjective value during intertemporal choice. Nat Neurosci 10:1625-1633.

Kuczenski R, Segal DS (1997) Effects of methylphenidate on extracellular dopamine, serotonin, and norepinephrine: Comparison with amphetamine. J Neurochem 68:2032-2037.

Lex A, Hauber W (2008) Dopamine D1 and D2 receptors in the nucleus accumbens core and shell mediate Pavlovian-instrumental transfer. Learn Mem 15:483-491.

Logue AW, Tobin H, Chelonis JJ, Wang RY, Geary N, Schachter S (1992) 
Cocaine decreases self control in rats: a preliminary report. Psychopharmacology 109:245-247.

Mai JK, Assheuer J, Paxinos G (2003) Atlas of the human brain, Ed 2. San Diego: Academic.

Mazur JE (1987) An adjusting procedure for studying delayed reinforcement. In: Quantitative analyses of behavior. V. The effect of delay and of intervening events on reinforcement value (Commons ML, Mazur JE, Nevin JA, Rachlin H, eds), pp 55-73. Hillsdale, NJ: Lawrence Erlbaum.

McClure SM, Laibson DI, Loewenstein G, Cohen JD (2004) Separate neural systems value immediate and delayed monetary rewards. Science 306: 503-507.

Mobini S, Chiang TJ, Al-Ruwaitea AS, Ho MY, Bradshaw CM, Szabadi E (2000) Effect of central 5-hydroxytryptamine depletion on inter-temporal choice: a quantitative analysis. Psychopharmacology (Berl) 149:313-318.

O'Sullivan SS, Evans, AH Lees AJ (2009) Dopamine dysregulation syndrome: an overview of its epidemiology, mechanisms and management. CNS Drugs 23:157-170.

Parkinson JA, Dalley JW, Cardinal RN, Bamford A, Fehnert B, Lachenal G, Rudarakanchana N, Halkerston KM, Robbins TW, Everitt BJ (2002) Nucleus accumbens dopamine depletion impairs both acquisition and performance of appetitive Pavlovian approach behaviour: implications for mesoaccumbens dopamine function. Behav Brain Res 137:149-163.

Pine A, Seymour B, Roiser JP, Bossaerts P, Friston KJ, Curran HV, Dolan RJ (2009) Encoding of marginal utility across time in the human brain. J Neurosci 29:9575-9581.

Pochon JB, Riis J, Sanfey AG, Nystrom LE, Cohen JD (2008) Functional imaging of decision conflict. J Neurosci 28:3468-3473.

Poulos CX, Parker JL, Le AD (1996) Dexfenfluramine and 8-OHDPAT modulate impulsivity in a delay-of-reward paradigm: implications for a correspondence with alcohol consumption. Behav Pharmacol 7:395-399.

Redish AD (2004) Addiction as a computation process gone awry. Science 306:1944-1947.

Richards JB, Seiden LS (1995) Serotonin depletion increases impulsive behavior in rats. Soc Neurosci Abstr 21:1693.

Richards JB, Sabol KE, de Wit H (1999) Effects of methamphetamine on the adjusting amount procedure, a model of impulsive behavior in rats. Psychopharmacology 146:432-439.

Robinson TE, Berridge KC (2008) The incentive sensitization theory of addiction: some current issues. Philos Trans R Soc Lond B Biol Sci 363: 3137-3146.

Sagvolden T, Sergeant JA (1998) Attention deficit/hyperactivity disorderfrom brain dysfunctions to behaviour. Behav Brain Res 94:1-10.

Seeman P, Madras BK (1998) Anti-hyperactivity medication: methylphenidate and amphetamine. Mol Psychiatry 3:386-396.

Seeman P, Madras B (2002) Methylphenidate elevates resting dopamine which lowers the impulse-triggered release of dopamine: a hypothesis. Behav Brain Res 130:79-83.
Seymour B, Dolan R (2008) Emotion, decision making, and the amygdala. Neuron 58:662-671.

Seymour B, Yoshida W, Dolan R (2009) Altruistic learning. Front Behav Neurosci 3:23.

Solanto MV (1998) Neuropsychopharmacological mechanisms of stimulant drug action in attention-deficit hyperactivity disorder: a review and integration. Behav Brain Res 94:127-152.

Solanto MV (2002) Dopamine dysfunction in AD/HD: integrating clinical and basic neuroscience research. Behav Brain Res 130:65-71.

Solanto MV, Abikoff H, Sonuga-Barke E, Schachar R, Logan GD, Wigal T, Hechtman L, Hinshaw S, Turkel E (2001) The ecological validity of delay aversion and response inhibition as measures of impulsivity in $\mathrm{AD} /$ HD: a supplement to the NIMH multimodal treatment study of AD/HD. J Abnorm Child Psychol 29:215-228.

Talairach J, Tournoux P (1988) Co-planar stereotaxic atlas of the human brain. Stuttgart: Thieme Publishing Group.

Talmi D, Seymour B, Dayan P, Dolan RJ (2008) Human Pavlovianinstrumental transfer. J Neurosci 28:360-368.

Tanaka SC, Doya K, Okada G, Ueda K, Okamoto Y, Yamawaki S (2004) Prediction of immediate and future rewards differentially recruits cortico-basal ganglia loops. Nat Neurosci 7:887-893.

van Gaalen MM, van Koten R, Schoffelmeer AN, Vanderschuren LJ (2006) Critical involvement of dopaminergic neurotransmission in impulsive decision making. Biol Psychiatry 60:66-73.

Voon V, Reynolds B, Brezing C, Gallea C, Skaljic M, Ekanayake V, Fernandez H, Potenza MN, Dolan RJ, Hallett M (2009) Impulsive choice and response in dopamine agonist-related impulse control behaviors. Psychopharmacology (Berl) 207:645-659.

Wade TR, de Wit H, Richards JB (2000) Effects of dopaminergic drugs on delayed reward as a measure of impulsive behavior in rats. Psychopharmacology 150:90-101.

Winstanley CA, Dalley JW, Theobald DE, Robbins TW (2003) Global 5-HT depletion attenuates the ability of amphetamine to decrease impulsive choice on a delay-discounting task in rats. Psychopharmacology 170: $320-331$

Winstanley CA, Dalley JW, Theobald DE, Robbins TW (2004a) Fractionating impulsivity: contrasting effects of central 5-HT depletion on different measures of impulsive behavior. Neuropsychopharmacology 29:13311343.

Winstanley CA, Theobald DE, Cardinal RN, Robbins TW (2004b) Contrasting roles of basolateral amygdala and orbitofrontal cortex in impulsive choice. J Neurosci 24:4718-4722.

Winstanley CA, Eagle DM, Robbins TW (2006) Behavioral models of impulsivity in relation to ADHD: translation between clinical and preclinical studies. Clin Psychol Rev 26:379-395.

Wogar MA, Bradshaw CM, Szabadi E (1993) Effects of lesions of the ascending 5-hydroxytryptaminergic pathways on choice between delayed reinforcers. Psychopharmacology 113:239-243. 